\title{
Quantum-mechanical ground state of dysprosium trichloride hexahydrate
}

\author{
E. Koutroumanou and A. K. Theophilou \\ Nuclear Research Center "Demokritos," Aghia Paraskevi Attikis, Greece \\ S. Thanos \\ Theoretical and Physical Chemistry Institute, National Hellenic Reserach Foundation, \\ Vasileos Constantinou 48, Athens 501/1, Greece \\ (Received 26 July 1985; revised manuscript received 21 April 1986)
}

\begin{abstract}
This paper deals with the quantum-mechanical ground state of dysprosium trichloride hexahydrate $\left(\mathrm{DyCl}_{3} \cdot 6 \mathrm{H}_{2} \mathrm{O}\right)$. The method used is that presented in the preceding companion paper. A discussion of the assumptions of the method for the extreme case of an Ising-like dipolar Hamiltonian is made, and the case of slightly modified correlation assumptions is examined. The quantummechanical ground state of $\mathrm{DyCl}_{3} \cdot 6 \mathrm{H}_{2} \mathrm{O}$ is found to be ferromagnetic with a strong magnetization component along the magnetic $z$ axis, and corresponding energy $-0.340 \mathrm{~K}$ per gram ion of $\mathrm{Dy}^{3+}$. The results found agree well with the existing experimental information about $\mathrm{DyCl}_{3} \cdot 6 \mathrm{H}_{2} \mathrm{O}$ and with the results of the semiclassical Luttinger-Tisza treatment.
\end{abstract}

\section{INTRODUCTION}

In this paper the method developed in the preceding paper $^{1}$ for the calculation of the ground state of dipolar crystals with two magnetic ions per unit cell is applied on the paramagnetic crystal of $\mathrm{DyCl}_{3} \cdot 6 \mathrm{H}_{2} \mathrm{O}$ (dysprosium trichloride hexahydrate).

The paramagnetic behavior of $\mathrm{DyCl}_{3} \cdot 6 \mathrm{H}_{2} \mathrm{O}$ is due to the dipole-dipole interaction between $\mathrm{Dy}^{3+}$ ions. Thus, in dealing with the magnetic properties of $\mathrm{DyCl}_{3} \cdot 6 \mathrm{H}_{2} \mathrm{O}$, one has to consider only the dysprosium ions. The spin (total angular momentum) of $\mathrm{Dy}^{3+}$ in the crystal field is $\frac{1}{2} .^{2,3}$ The two dysprosium ions in the crystallographic unit cell are magnetically equivalent.

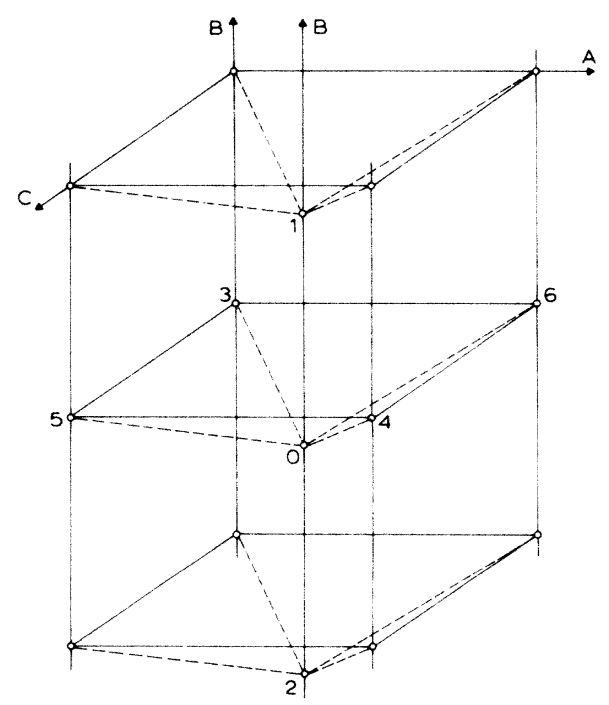

FIG. 1. Crystal structure of $\mathrm{DyCl}_{3} \cdot 6 \mathrm{H}_{2} \mathrm{O}$. Only the magnetic ions $\mathrm{Dy}^{3+}$ are indicated. Two monoclinic unit cells are shown, each containing two $\mathrm{Dy}^{3+}$ ions.
The crystal structure of $\mathrm{DyCl}_{3} \cdot 6 \mathrm{H}_{2} \mathrm{O}$ is monoclinic, with point group $C_{2 h}$ and space group $P 2 / \mathrm{m}^{4}$ The lattice of the dysprosium ions is shown in Fig. 1. $B$ is the monoclinic symmetry axis. The cell parameters are ${ }^{4}$

$$
A=9.61 \AA, B=6.49 \AA, C=7.87 \AA \text {, }
$$

and

$$
\beta=93^{\circ} 40^{\prime} \text {. }
$$

The position vector specifying the site of the one dysprosium ion relative to the other in the unit cell, is

$$
\mathbf{d}=\frac{1}{2} \mathbf{a}_{1}+0.3042 \mathbf{a}_{2}+\frac{1}{2} \mathbf{a}_{3} \text {. }
$$

In the following we shall use as a coordinate system the

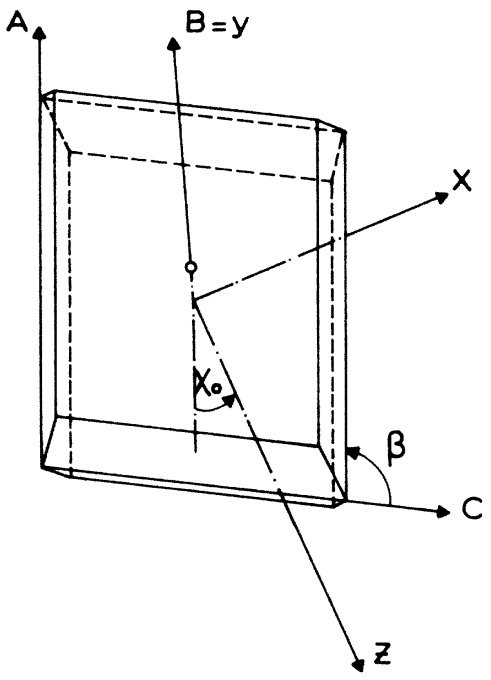

FIG. 2. The principal axes $x, y, z$ of the $g$ tensor of the Dy ${ }^{3+}$ ions and the crystallographic axes $A, B, C$ of the $\mathrm{DyCl}_{3} \cdot 6 \mathrm{H}_{2} \mathrm{O}$ crystal. 


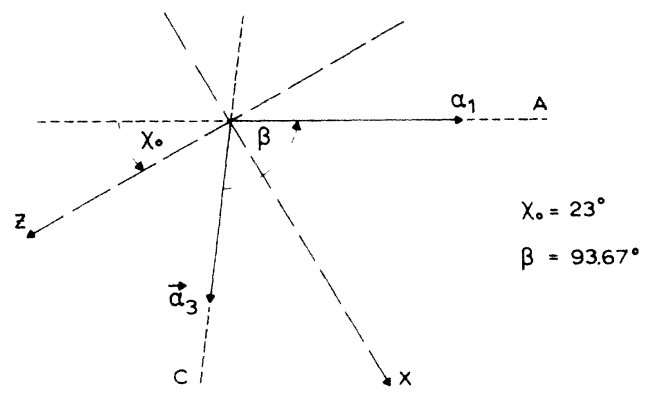

FIG. 3. The crystallographic plane $A C$ of $\mathrm{DyCl}_{3} \cdot 6 \mathrm{H}_{2} \mathrm{O}$ and the magnetic axes $z$ and $x$. The magnetic axis $y$ coincides with the crystallographic axis $B$ and is vertical to the plane of the figure.

system of the magnetic axes, i.e., the principal axes of the $g$ tensor of the dysprosium ions. The magnetic $y$ axis coincides with the crystallographic axis $B$, but the other magnetic axes are not simply related to the crystal axes. ${ }^{2,3}$ The two systems of axes are presented in Figs. 2 and 3. We used the $g$ values of Ref. 3:

$$
g_{\|}=g_{z}=16.52 \text { and } g_{\perp}=1.76
$$

As the distances between the magnetic ions are large, and the $4 f$ orbitals which give rise to their spin are localized, exchange interactions are negligible.

The experimental results of Lagendijk and Huiskamp ${ }^{2}$ suggest that $\mathrm{DyCl}_{3} \cdot 6 \mathrm{H}_{2} \mathrm{O}$ is ferromagnetic at temperatures below $T_{c}=0.289 \mathrm{~K}$. The ground-state energy obtained from their specific-heat data with an exponential extrapolation $^{2}$ is estimated to be between $-0.32 \mathrm{~K}$ (Ref. 2) and $-0.35 \mathrm{~K}$ (Ref. 5) per gram ion of $\mathrm{Dy}^{3+}$.

Lagendijk and co-workers ${ }^{2,5}$ as well as Niemeijer and
Blöte ${ }^{6}$ calculated the ground state of $\mathrm{DyCl}_{3} \cdot 6 \mathrm{H}_{2} \mathrm{O}$, using the classical method of Luttinger and Tisza. ${ }^{7}$ They derived a ferromagnetic ground state with classical magnetic moments oriented along the magnetic $z$ axis, and a ground-state energy of $-0.338 \mathrm{~K}$ per gram ion of $\mathrm{Dy}^{3+}$.

$\mathrm{ErCl}_{3} \cdot 6 \mathrm{H}_{2} \mathrm{O}$ and $\mathrm{GdCl}_{3} \cdot 6 \mathrm{H}_{2} \mathrm{O}$ have similar crystal structures to $\mathrm{DyCl}_{3} \cdot 6 \mathrm{H}_{2} \mathrm{O}$. The classical ground state of $\mathrm{ErCl}_{3} \cdot 6 \mathrm{H}_{2} \mathrm{O}$ was found by Felsteiner ${ }^{8}$ to be ferromagnetic. This is not the case with the ground state of $\mathrm{GdCl}_{3} \cdot 6 \mathrm{H}_{2} \mathrm{O}$ which, according to Misra and Felsteiner, ${ }^{9}$ is antiferromagnetic. However, in this crystal the exchange interactions are not negligible.

Section II of this paper deals with the Hamiltonian of the magnetic ions of $\mathrm{DyCl}_{3} \cdot 6 \mathrm{H}_{2} \mathrm{O}$. In Sec. III we comment on the assumptions of the Niemeijer-Meijer method for the extreme case of an Ising-like dipolar Hamiltonian. In Sec. IV we present our results for the ground state of DyCl $3 \cdot 6 \mathrm{H}_{2} \mathrm{O}$ and their relation to the experimental data.

For the sake of completeness, we give in Table I the lattice sums in the coordinate system of the magnetic axes, derived from the corresponding calculations of Niemeijer and Blöte. ${ }^{6}$

\section{THE EFFECTIVE HAMILTON OF DYSPROSIUM IONS}

The Hamiltonian of the system of dysprosium ions of the dipolar crystal $\mathrm{DyCl}_{3} \cdot 6 \mathrm{H}_{2} \mathrm{O}$ is the typical dipoledipole interaction Hamiltonian: 1,10

$$
\mathscr{H}=\frac{1}{2} \sum_{\substack{i, j \\ i \neq j}} \frac{1}{\left|\mathbf{R}_{i j}\right|^{3}}\left[\boldsymbol{\mu}_{i} \cdot \boldsymbol{\mu}_{j}-3 \frac{\left(\boldsymbol{\mu}_{i} \cdot \mathbf{R}_{i j}\right)\left(\boldsymbol{\mu}_{j} \cdot \mathbf{R}_{i j}\right)}{\left|\mathbf{R}_{i j}\right|^{2}}\right)
$$

TABLE I. The lattice sums $A^{\alpha \beta}(\mathbf{R}), \alpha, \beta=x, y, z, \mathbf{R} \in C$, in units of $\mathrm{K}$ per gram ion of $\mathrm{Dy}^{3+}$, for the choice $\Gamma^{\prime}=\Gamma^{2}$ in the coordi-

\begin{tabular}{|c|c|c|c|c|c|c|}
\hline $\mathbf{R}$ & $A_{0}^{x x}(\mathbf{R})$ & $A_{O}^{y y}(\mathbf{R})$ & $A_{0}^{z z}(\mathbf{R})$ & $A_{0}^{x y}(\mathbf{R})$ & $A_{0}^{x z}(\mathbf{R})$ & $A_{0}^{y z}(\mathbf{R})$ \\
\hline 0 & -0.00019 & -0.00052 & -0.00550 & 0 & -0.00085 & 0 \\
\hline $\boldsymbol{\alpha}_{1}$ & -0.000154 & 0.00051 & 0.02276 & 0 & -0.01128 & 0 \\
\hline $\boldsymbol{\alpha}_{2}$ & 0.00141 & -0.00372 & 0.13485 & 0 & -0.00078 & 0 \\
\hline $\boldsymbol{\alpha}_{1}+\boldsymbol{\alpha}_{2}$ & -0.00060 & -0.00045 & 0.02497 & 0 & -0.00552 & 0 \\
\hline$\alpha_{3}$ & 0.00005 & 0.00020 & -0.09021 & 0 & 0.00545 & 0 \\
\hline $\boldsymbol{\alpha}_{1}+\boldsymbol{\alpha}_{3}$ & -0.00039 & 0.00009 & -0.04172 & 0 & 0.00159 & 0 \\
\hline$\alpha_{2}+\alpha_{3}$ & 0.00005 & -0.00019 & -0.05644 & 0 & 0.00358 & 0 \\
\hline $\boldsymbol{\alpha}_{1}+\boldsymbol{\alpha}_{2}+\boldsymbol{\alpha}_{3}$ & -0.00029 & -0.00008 & -0.03436 & 0 & 0.00128 & 0 \\
\hline d & -0.00130 & 0.00035 & 0.01557 & -0.00066 & 0.00881 & 0.00313 \\
\hline $\boldsymbol{\alpha}_{1}+\mathbf{d}$ & 0.00031 & 0.00030 & -0.12154 & 0.00013 & -0.00482 & 0.00519 \\
\hline$\alpha_{2}+d$ & -0.00050 & -0.00042 & 0.01358 & 0.00047 & 0.00383 & -0.00225 \\
\hline$\alpha_{1}+\alpha_{2}+d$ & 0.00018 & -0.00035 & -0.05353 & -0.00010 & -0.00234 & -0.00391 \\
\hline$\alpha_{3}+d$ & 0.00031 & 0.00030 & -0.12154 & -0.00013 & -0.00482 & -0.00519 \\
\hline$\alpha_{1}+\alpha_{3}+d$ & -0.00130 & 0.00035 & 0.01557 & 0.00066 & 0.00881 & -0.00313 \\
\hline$\alpha_{2}+\alpha_{3}+d$ & 0.00018 & -0.00035 & -0.05353 & 0.00010 & -0.00234 & 0.00391 \\
\hline$\alpha_{1}+\alpha_{2}+\alpha_{3}+d$ & -0.00050 & -0.00042 & 0.01358 & -0.00047 & 0.00383 & 0.00225 \\
\hline
\end{tabular}
nate system of the magnetic axes. 


$$
\begin{aligned}
& \left\langle\Psi\left|S^{\alpha}(\mathbf{R}+\mathbf{t})\right| \Psi\right\rangle=\left\langle\Psi\left|S^{\alpha}(\mathbf{R})\right| \Psi\right\rangle \\
& \left\langle\Psi\left|S^{\alpha}(\mathbf{R}+\mathbf{t}) S^{\beta}\left(\mathbf{R}+\mathbf{t}^{\prime}\right)\right| \Psi\right\rangle=\left\langle\Psi\left|S^{\alpha}(\mathbf{R})\right| \Psi\right\rangle\left\langle\Psi\left|S^{\beta}(\mathbf{R})\right| \Psi\right\rangle, \quad \mathbf{t} \neq \mathbf{t}^{\prime} \\
& \left\langle\Psi\left|S^{\alpha}(\mathbf{R}+\mathbf{t}) S^{\beta}\left(\mathbf{R}^{\prime}+\mathbf{t}^{\prime}\right)\right| \Psi\right\rangle=\left\langle\Psi\left|S^{\alpha}(\mathbf{R}) S^{\beta}\left(\mathbf{R}^{\prime}\right)\right| \Psi\right\rangle, \quad \mathbf{R} \neq \mathbf{R}^{\prime},
\end{aligned}
$$

where $\alpha, \beta=x, y, z, \mathbf{R}, \mathbf{R}^{\prime} \in C$, and $\mathbf{t}, \mathbf{t}^{\prime} \in \Gamma^{\prime}$ (see Ref. 1), lead to the effective eight-ion cluster Hamiltonian $H$ :

$$
\begin{aligned}
H= & H^{\prime}+H_{\delta}, \\
H^{\prime} & =\frac{1}{2} \sum_{\alpha, \beta=x, y, z} \sum_{R \in C} \sum_{\mathbf{R}^{\prime} \in C} A^{\alpha \beta}\left(\mathbf{R}-\mathbf{R}^{\prime}\right) S^{\alpha}(\mathbf{R}) S^{\beta}\left(\mathbf{R}^{\prime}\right), \\
H_{\delta}= & \frac{1}{2} \sum_{\alpha, \beta=x, y, z} A^{\alpha \beta} \sum_{\mathbf{R} \in C}\left\langle\Psi\left|S^{\alpha}(\mathbf{R})\right| \Psi\right\rangle S^{\beta}(\mathbf{R}) \\
& -\sum_{\alpha=x, y, z} A^{\alpha \alpha}(0),
\end{aligned}
$$

where the quantities $A^{\alpha \beta}(\mathbf{R})$ are defined in Ref. 1. Because of the nonlinearity of $H_{\delta}$, we find the eigenvalues and eigenstates of the linear operator $H^{\prime}$, and treat $H_{\delta}$ by using perturbation theory (see Ref. 1).

Since the classical solution for the ground state of $\mathrm{DyCl}_{3} \cdot 6 \mathrm{H}_{2} \mathrm{O}$ is ferromagnetic, by the argument presented in Ref. 1, we can choose any axis as the direction of single periodicity. Thus, we made three different choices of periodicity specified by (A), (B), and (C), which indicate the crystallographic axis chosen as the direction of single periodicity in each case. The subgroup $\Gamma^{\prime}$ of lattice translations ${ }^{1}$ we consider in each case is as follows: in case (A),

$$
\Gamma^{\prime}=\Gamma_{1}=\left(\mathbf{t}=n_{1} \boldsymbol{\alpha}_{1}+2 n_{2} \boldsymbol{\alpha}_{2}+2 n_{3} \boldsymbol{\alpha}_{3}\right),
$$

in case $(\mathrm{B})$,

$$
\Gamma^{\prime}=\Gamma_{2}=\left(\mathbf{t}=2 n_{1} \alpha_{1}+n_{2} \alpha_{2}+2 n_{3} \alpha_{3}\right),
$$

and in case $(\mathrm{C})$,

$$
\Gamma^{\prime}=\Gamma_{3}=\left(\mathbf{t}=2 n_{1} \boldsymbol{\alpha}_{1}+2 n_{2} \boldsymbol{\alpha}_{2}+n_{3} \boldsymbol{\alpha}_{3}\right) .
$$

The coefficients $A^{\alpha \beta}(\mathbf{R})$ needed in each case, can be found from the lattice sums of Table I by addition, e.g., in case $(\mathrm{A})$ :

$$
A_{1}^{\alpha \beta}(\mathbf{R})=A_{0}^{\alpha \beta}(\mathbf{R})+A_{0}^{\alpha \beta}\left(\mathbf{R}+\alpha_{1}\right) .
$$

Due to the strong anisotropy $g_{z}=g_{\|} \gg g_{1}$, and the absence of exchange interactions, we expect the interactions between the dysprosium ions to be Ising-like. Hence the dipolar Hamiltonian (1) of $\mathrm{DyCl}_{3} \cdot 6 \mathrm{H}_{2} \mathrm{O}$ can be approximated by the Ising Hamiltonian:

$$
\mathscr{H}_{\mathbf{z}}=\frac{1}{2} \sum_{\substack{i, j \\ i \neq j}} J^{z z}\left(\mathbf{R}_{i}-\mathbf{R}_{j}\right) S^{z}\left(\mathbf{R}_{i}\right) S^{z}\left(\mathbf{R}_{j}\right),
$$

where

$$
J^{z z}(\mathbf{R})=m_{B}^{2} g_{\|}^{2} \frac{|\mathbf{R}|^{2}-3\left(R^{z}\right)^{2}}{|\mathbf{R}|^{5}}, \quad \mathbf{R} \neq 0 .
$$

The linear operator $H^{\prime}$ that we have to diagonalize, can also be approximated by the Ising operator:

$$
H_{z}^{\prime}=\frac{1}{2} \sum_{\mathbf{R} \in C} \sum_{\mathbf{R}^{\prime} \in C} A^{z z}\left(\mathbf{R}-\mathbf{R}^{\prime}\right) S^{z}(\mathbf{R}) S^{z}\left(\mathbf{R}^{\prime}\right) .
$$

This is the simplest possible case to which we can apply the Niemeijer-Meijer quantum method, ${ }^{1,10}$ so we were able to test our assumptions [Eqs. (2), (3), and (4)] for this special case.

\section{DISCUSSION ON THE ASSUMPTIONS}

If we apply the quantum-mechanical method developed in the preceding paper, ${ }^{1}$ under the Niemeijer-Meijer correlation assumptions, i.e., (2), (3), and (4), we will find among the 256 solutions 14 eigenstates of $H_{z}^{\prime}$ of the following form:

$$
\begin{aligned}
& \left|\Phi_{\mathbf{k}_{0}^{+}}^{+}\left(\mathbf{k}^{ \pm}\right)\right\rangle=\frac{1}{\sqrt{2}}\left[\prod_{\mathbf{R} \in C}\left|\chi_{\mathbf{R}}^{\mathbf{k}^{ \pm}}\left(\mathbf{S}_{\mathbf{R}}\right)\right\rangle+S \prod_{\mathbf{R} \in C}\left|\chi_{\mathbf{R}}^{\mathbf{k}^{ \pm}}\left(\mathbf{S}_{\mathbf{R}}\right)\right\rangle\right], \\
& \left|\Phi_{\mathbf{k}^{ \pm}}^{- \pm}\left(\mathbf{k}^{ \pm}\right)\right\rangle=\frac{1}{\sqrt{2}}\left[\prod_{\mathbf{R} \in C}\left|\chi_{\mathbf{R}}^{\mathbf{k}^{ \pm}}\left(\mathbf{S}_{\mathbf{R}}\right)\right\rangle-S \prod_{\mathbf{R} \in C}\left|\chi_{\mathbf{R}}^{\mathbf{k}^{ \pm}}\left(\mathbf{S}_{\mathbf{R}}\right)\right\rangle\right]
\end{aligned}
$$

with $\mathbf{k}^{ \pm} \neq \mathbf{k}_{0}^{+}$, where $S$ is the spin-flip operator

$$
S=\prod_{R \in C}\left[S^{+}(\mathbf{R})+S^{-}(\mathbf{R})\right] .
$$

The state $\left.\left.\mid \chi_{\mathbf{R}}^{\mathbf{k}} \pm \mathbf{S}_{\mathbf{R}}\right)\right\rangle$ is the spin state of the ion at site $\mathbf{R}$ of the basic cell. It is an eigenstate of $S^{z}(\mathbf{R})$ with eigenvalue $\frac{1}{2}$ times the value of the $\mathbf{k}^{ \pm}$irreducible representation for the lattice translation or inversion transforming site 0 to site $\mathbf{R}$. We would like to mention again that these representations are one dimensional.

States $\left|\Phi_{\mathbf{k}_{0}^{+}}^{+}\left(\mathbf{k}^{ \pm}\right)\right\rangle$and $\left|\Phi_{\mathbf{k}^{ \pm}}^{- \pm}\left(\mathbf{k}^{ \pm}\right)\right\rangle$are both antiferromagnetic:

$$
\begin{aligned}
& \left\langle\Phi_{\mathbf{k}_{0}^{+}}^{+}\left(\mathbf{k}^{ \pm}\right)\left|S^{z}(\mathbf{R})\right| \Phi_{\mathbf{k}_{0}^{+}}^{+}\left(\mathbf{k}^{ \pm}\right)\right\rangle \\
& =\left\langle\Phi_{\mathbf{k}^{ \pm}}^{-}\left(\mathbf{k}^{ \pm}\right)\left|S^{z}(\mathbf{R})\right| \Phi_{\mathbf{k}^{ \pm}}^{-}\left(\mathbf{k}^{ \pm}\right)\right\rangle=0 .
\end{aligned}
$$

States $\left|\Phi_{\mathbf{k}_{0}^{+}}^{+}\left(\mathbf{k}^{ \pm}\right)\right\rangle$and $\left|\Phi_{\mathbf{k}^{ \pm}}^{-}\left(\mathbf{k}^{ \pm}\right)\right\rangle$are degenerate eigenstates of the operator $H_{z}^{\prime}$ with eigenvalue $\lambda_{\mathbf{k} \pm}^{z}$, equal to the energy of the corresponding classical solution $\left|\xi_{k^{ \pm}}^{z}\right\rangle$. These states are exact eigenstates of the nonlinear Hamiltonian:

$$
H_{z}=H_{z}^{\prime}-A^{z z}(0)+\frac{1}{2} A^{z z}(0) \sum_{\mathbf{R} \in C}\left\langle\Psi\left|S^{z}(\mathbf{R})\right| \Psi\right\rangle S^{z}(\mathbf{R})
$$

with eigenvalue equal to $\lambda_{\mathrm{k} \pm}^{z}-A^{z z}(0)$. However, they have the disadvantage that they do not satisfy conditions 
(3) and (4) simultaneously (see proof in Appendix A), i.e., they are not acceptable solutions. The physical conclusion is that it is not possible to have the spin value at site $\mathbf{R}$ not correlated to that of its next-nearest neighbor, while there is full correlation with its nearest neighbors.

It is to be noted that the $\left|\Phi_{\mathbf{k}_{0}^{+}}^{+}\left(\mathbf{k}^{ \pm}\right)\right\rangle$and $\left|\Phi_{\mathbf{k}^{ \pm}}^{-}\left(\mathbf{k}^{ \pm}\right)\right\rangle$ are degenerate states of the linear operator $H_{z}^{\prime}$. The state derived by their sum is a product of single-particle states. This state, which does not belong to a particular irrep of our group, satisfies the conditions (2), (3), and (4). This is a very welcome state as it corresponds to a classical spin state, and is an exact eigenstate of the nonlinear operator $H_{z}$ [see Eq. (13)] with an eigenvalue that is the same as that of $H_{z}^{\prime}$.

Since the assumption, that spins at lattice sites differing by a sublattice translation are uncorrelated, does not derive from any fundamental principle, we thought of searching for self-consistent solutions when other correlation assumptions are made. Thus we tried the case that the spins at $\mathbf{R}$ and $\mathbf{R}+\mathbf{t}$ are fully correlated. Then instead of condition (3) we have the following condition:

$$
\left\langle\Psi\left|S^{z}(\mathbf{R}+\mathbf{t}) S^{z}\left(\mathbf{R}+\mathbf{t}^{\prime}\right)\right| \Psi\right\rangle=\frac{1}{4} \text {. }
$$

Conditions (2), (4), and (14) are derivable from wave functions of the following form (see Appendix B):

$$
|\Psi\rangle=\sum_{l=1}^{256} c_{l} \prod_{\mathbf{t} \in \Gamma^{\prime}} \prod_{\mathbf{R} \in c}\left|\chi_{\mathbf{R}}^{l}\left(\mathbf{S}_{\mathbf{R}+\mathbf{t}}\right)\right\rangle,
$$

where the coefficients $c_{l}$ obey the normalization condition $\sum_{l=1}^{256}\left|c_{l}\right|^{2}=1$ and the state $\left|\chi_{\mathbf{R}}^{l}\left(\mathbf{S}_{\mathbf{R}+t}\right)\right\rangle$ is the one-spin state of the ion at $\mathbf{R}+\mathbf{t}$, depends only on $\mathbf{R}$, and is an eigenstate of $S^{z}(\mathbf{R}+\mathbf{t})$ with eigenvalue $\pm \frac{1}{2}$. These wave functions allow nonzero correlation between any spin pair.

The resulting equation for the extrema of $\left\langle\Psi\left|\mathscr{H}_{z}\right| \Psi\right\rangle$ is $H_{z}^{\prime}|\Psi\rangle=E_{z}|\Psi\rangle$. The resulting $H_{z}^{\prime}$ is the same as that defined by Eq. (9) (see Appendix B). Thus no nonlinear term appears.

All resulting solutions are consistent with the assumptions (2), (4), and (14). In addition, the states corresponding to the extrema are single products of one-spin states, while because of the degeneracies, linear combinations of such states appear as solutions.

\section{RESULTS}

We performed our calculations for all cases (A), (B), and (C) (see Sec. II). Both sets of correlation assumptions were used for each case.

The two sets of correlation assumptions yield almost the same approximate eigenstates, i.e., the eigenstates of the linear operator $H^{\prime}$ [see Eq. (6)]. The only difference is that, when we assume condition (3), we substitute the eigenstates of the form (10) by their linear combinations, although the latter do not belong to a particular irrep of the translation group. As is well known, nonlinear effective Hamiltonians possess eigenstates which do not belong to a single irrep of the invariance group of the exact Hamiltonian.

We also found almost the same energy values with the two sets of correlation assumptions for the lowest-energy solutions. The correction term for the nonlinear Hamiltonian was very small and positive for the solutions compatible with the correlation assumptions (see Table II). This agreement is due to the fact that the dipolar Hamiltonian of $\mathrm{DyCl}_{3} \cdot 6 \mathrm{H}_{2} \mathrm{O}$ is almost an Ising Hamiltonian.

According to our results for all three cases of periodicity conditions (A), (B), and (C), the dipolar crystal $\mathrm{DyCl}_{3} \cdot 6 \mathrm{H}_{2} \mathrm{O}$ is ferromagnetic in its ground state, with strong magnetization component along the magnetic $z$ axis. The ground state is found doubly degenerate, belonging to the identity $\left(\mathbf{k}_{0}^{+}\right)$irrep, with energy $-0.340 \mathrm{~K}$ per gram ion of $\mathrm{Dy}^{3+}$. The semiclassical treatment gives the value $-0.338 \mathrm{~K}$. Thus, as in the case of cerium magnesium nitrate $(\mathrm{CMN})$, the semiclassical treatment gives quick and accurate results when no magnetic field is present. $^{11}$

Contrary to the ground state, the next lower-energy state is found to be different. It is an antiferromagnetic, doubly degenerate solution, with energy $-0.319 \mathrm{~K}$ in case (A), $-0.317 \mathrm{~K}$ in case $(\mathrm{C})$, and $-0.261 \mathrm{~K}$ in case $(\mathrm{B})$.

Our theoretical calculations agree with the experimental results of Lagendijk and Huiskamp, who find a ferromagnetic ground state with energy in the range $-0.32 \mathrm{~K}$ to $-0.35 \mathrm{~K}$ per gram ion. ${ }^{2,5}$

TABLE II. The two lowest energy states calculated for cases (A), (B), and (C). The correction energies when condition (3) is assumed are indicated by $\Delta E$. The energy units are $\mathrm{K}$ per gram ion of $\mathrm{Dy}^{3+}$. By 0 we indicate the ground state, which in all cases is found to be ferromagnetic, doubly degenerate, and transforming according to the identity irreducible representations. The next lower energy state is indicated by 1 . It is antiferromagnetic, doubly degenerate, and is a linear combination of $\mathbf{k}^{ \pm}$states indicated in parentheses in the third column.

\begin{tabular}{cccccc}
\hline \hline Case & State & $\begin{array}{c}\text { Irreducible } \\
\text { representations }\end{array}$ & $E$ & $\Delta E$ & $|\Delta E| /|E|$ \\
\hline (A) & 0 & $\mathbf{k}_{0}^{+}$ & -0.33935 & +0.00269 & 0.8 \\
& 1 & $\left(\mathbf{k}_{0}^{+}, \mathbf{k}_{2}^{+}\right)$ & -0.31874 & +0.00170 & 0.5 \\
(B) & 0 & $\mathbf{k}_{0}^{+}$ & -0.34006 & +0.00304 & 0.9 \\
& 1 & $\left(\mathbf{k}_{0}^{+}, \mathbf{k}_{2}^{-}\right)$ & -0.26057 & +0.00337 & 1.3 \\
& & & & \\
(C) & 0 & $\mathbf{k}_{0}^{+}$ & -0.33754 & +0.00082 & 0.2 \\
& 1 & $\left(\mathbf{k}_{0}^{+}, \mathbf{k}_{2}^{+}\right)$ & -0.31651 & +0.00045 & 0.1 \\
\hline \hline
\end{tabular}




\section{APPENDIX A}

We shall show that a cluster state $|\Phi\rangle$ as specified by Eq. (10) gives correlations not compatible with a total crystal state $|\Psi\rangle$ obeying the conditions given by Eqs. (3) and (4).

Let $|\Psi\rangle$ be a total crystal state, i.e., a state describing all magnetic ions of the crystal, that corresponds to the above cluster state $|\Phi\rangle$. This correspondence specifies that states $|\Phi\rangle$ and $|\Psi\rangle$ give the same expectation values of the spin operators and the same spin correlations, in the cluster, and $|\Psi\rangle$ gives the expectation values of the spins and spin correlations for magnetic ions in different clusters, as specified by conditions (2), (3), and (4). Consequently, $|\Psi\rangle$ gives the same energy per cluster, as the expectation value of the effective Hamiltonian [see Eqs. (5), (6), and (7)] for the cluster state $|\Phi\rangle$.

Then, according to conditions (3) and (4), state $|\Psi\rangle$ has the following properties [see Eqs. (10) and (12)]:

$$
\begin{aligned}
& \left\langle\Psi\left|S^{\mathbf{z}}(\mathbf{R}+\mathbf{t}) S^{z}\left(\mathbf{R}+\mathbf{t}^{\prime}\right)\right| \Psi\right\rangle=0, \quad \mathbf{t} \neq \mathbf{t}^{\prime} \\
& \left\langle\Psi\left|S^{z}(\mathbf{R}+\mathbf{t}) S^{z}\left(\mathbf{R}^{\prime}+\mathbf{t}^{\prime}\right)\right| \Psi\right\rangle= \pm \frac{1}{4}, \quad \mathbf{R} \neq \mathbf{R}^{\prime},
\end{aligned}
$$

where $\mathbf{R}, \mathbf{R}^{\prime} \in C$ and $\mathbf{t}, \mathbf{t}^{\prime} \in \Gamma^{\prime}$.

The spin correlations between three particular sites $\mathbf{R}_{0}=0, \mathbf{R}_{1} \in C$, and $\mathbf{R}_{2} \in \Gamma^{\prime}$, given by state $|\Psi\rangle$, are

$$
\begin{aligned}
& \left\langle\Psi\left|S^{z}\left(\mathbf{R}_{0}\right) S^{z}\left(\mathbf{R}_{2}\right)\right| \Psi\right\rangle=0, \\
& \left\langle\Psi\left|S^{z}\left(\mathbf{R}_{0}\right) S^{z}\left(\mathbf{R}_{1}\right)\right| \Psi\right\rangle=\left\langle\Psi\left|S^{z}\left(\mathbf{R}_{2}\right) S^{z}\left(\mathbf{R}_{1}\right)\right| \Psi\right\rangle=+\frac{1}{4} .
\end{aligned}
$$

Any total crystal state $|\Psi\rangle$ has the form

$$
\begin{aligned}
& |\Psi\rangle=\sum_{l} c_{l}\left|\Psi_{l}\right\rangle, \\
& \left|\Psi_{l}\right\rangle=\prod_{\mathbf{t} \in \Gamma^{\prime}} \prod_{\mathbf{R} \in C}\left|\chi_{\mathbf{R}+\mathbf{t}}^{l}\left(\mathbf{S}_{\mathbf{R}+\mathbf{t}}\right)\right\rangle,
\end{aligned}
$$

where $\left|\chi_{\mathbf{R}+\mathrm{t}}\left(\mathbf{S}_{\mathbf{R}+\mathbf{t}}\right)\right\rangle$ is an eigenstate of $S^{\mathbf{z}}(\mathbf{R}+\mathbf{t})$ with eigenvalue $\mu_{\mathrm{R}+\mathrm{t}}^{l}= \pm \frac{1}{2}$, as the $\left|\Psi_{l}\right\rangle$ form a complete orthonormal set of states for the crystal.

Equation (A4) implies that, for all $l$ with $c_{l} \neq 0$,

$$
\mu_{\mathbf{R}_{0}}^{l} \mu_{\mathbf{R}_{1}}^{l}=\mu_{\mathbf{R}_{2}}^{l} \mu_{\mathbf{R}_{1}}^{l}=+\frac{1}{4} \text {. }
$$

Hence, for all $l$ with $c_{l} \neq 0$,

$$
\mu_{\mathbf{R}_{0}}^{l}=\mu_{\mathbf{R}_{2}}^{l},
$$

which contradicts Eq. (A3).

Thus, one deduces that relations (A1) and (A2) cannot hold simultaneously. Then, according to the hypotheses (3) and (4), the cluster states $\left|\Phi_{\mathbf{k}_{0}^{+}}^{+}\left(\mathbf{k}^{ \pm}\right)\right\rangle$and $\left|\boldsymbol{\Phi}_{\mathbf{k}^{ \pm}}^{-}\left(\mathbf{k}^{ \pm}\right)\right\rangle$ [see Eq. (10)] correspond to no total crystal state and therefore they should be rejected.

\section{APPENDIX B}

In this appendix we shall determine the form of the solutions and derive the cluster equations when condition (3) is substituted by condition (14). We shall first prove that a total crystal state $|\Psi\rangle$ that fulfills the condition given by Eq. (14) is always a state of the form (15). The converse is also true, i.e., if $|\Psi\rangle$ is of the form (15), then it satisfies conditions (2), (4), and (14).

By introducing into condition (14) the explicit form of $|\Psi\rangle$ given by Eqs. (A5) and (A6) of Appendix A, we get

$$
\sum_{l}\left|c_{l}\right|^{2} \mu_{\mathrm{R}+\mathrm{t}}^{l} \mu_{\mathrm{R}+\mathrm{t}^{\prime}}^{l}=\frac{1}{4} \text {. }
$$

Since $\sum_{l}\left|c_{l}\right|^{2}=1$ and $\mu_{\mathrm{R}+\mathrm{t}}^{l}= \pm \frac{1}{2}$, it follows that

$$
\mu_{\mathrm{R}+\mathrm{t}}^{l}=\mu_{\mathrm{R}+\mathrm{t}^{\prime}}^{l}
$$

for every $l$ with $c_{l} \neq 0, \mathbf{R} \in C$, and $\mathbf{t}, \mathbf{t}^{\prime} \in \Gamma^{\prime}$. Hence $\mu_{\mathrm{R}+\mathrm{t}}^{l}$ does not depend on $t$. As $\left|\chi_{\mathbf{R}+\mathbf{t}}^{l}\left(\mathbf{S}_{\mathbf{R}+\mathrm{t}}\right)\right\rangle$ is uniquely determined by the eigenvalue of $S^{z}(\mathbf{R}+\mathbf{t})$, it follows that it does not depend on $t$. Thus state $|\Psi\rangle$ is reduced to the form of (15).

Conversely, for a total crystal state $|\Psi\rangle$ of the form of (15), it is easily seen that

$$
\begin{aligned}
& \left\langle\Psi\left|S^{z}(\mathbf{R}+\mathbf{t})\right| \Psi\right\rangle=\sum_{l=1}^{256}\left|c_{l}\right|^{2} \mu_{\mathrm{R}}^{l}, \\
& \left\langle\Psi\left|S^{z}(\mathbf{R}+\mathbf{t}) S^{z}\left(\mathbf{R}^{\prime}+\mathbf{t}^{\prime}\right)\right| \Psi\right\rangle=\sum_{l=1}^{256}\left|c_{l}\right|^{2} \mu_{\mathrm{R}^{\prime}}^{l} \mu_{\mathrm{R}^{\prime}}^{l},
\end{aligned}
$$

therefore conditions (2), (4), and (14) hold.

For any cluster state $|\Phi\rangle$, we can construct the corresponding total crystal state $|\Psi\rangle$ that gives the same expectation values of the spin operators and the same spin correlations as $|\Phi\rangle$ in the cluster, and the ones specified by conditions (2), (4), and (14), for magnetic ions in different clusters. The construction procedure is very simple.

The cluster states

$$
\left|\Phi^{l}\right\rangle=\prod_{\mathbf{R} \in C}\left|\chi_{\mathbf{R}}^{l}\left(\mathbf{S}_{\mathbf{R}}\right)\right\rangle
$$

form an orthonormal basis for the cluster state space. Thus every solution of our method can be written in the form:

$$
|\Phi\rangle=\sum_{l=1}^{256} a_{l}\left|\Phi^{l}\right\rangle .
$$

A total crystal state $|\Psi\rangle$ given by Eq. (15) with $c_{l}=a_{l}$ corresponds to every solution $|\Phi\rangle$. This $|\Psi\rangle$ has the properties described above, and consequently gives the same energy per cluster, as the expectation value of the linear part of the effective Hamiltonian [see Eq. (9)] for the solution $|\Phi\rangle$ :

$$
\frac{1}{N}\left\langle\Psi\left|\mathscr{H}_{z}\right| \Psi\right\rangle=\left\langle\Phi\left|H_{z}^{\prime}\right| \Phi\right\rangle
$$

i.e., the cluster Hamiltonian is now the linear operator $H_{z}^{\prime}$. Furthermore, if $H_{z}^{\prime}|\Phi\rangle=E_{z}|\Phi\rangle$, then

$$
\frac{1}{N} \mathscr{H}_{z}|\Psi\rangle=E_{z}|\Psi\rangle \text {, }
$$

i.e., $|\Psi\rangle$ is an exact eigenstate of the total Hamiltonian $\mathscr{H}_{z}$ with the same eigenvalue $E_{z}$.

Therefore, when our Hamiltonian is exactly Ising (and $s=\frac{1}{2}$ ), we can apply the method of the preceding paper, with the only modification that we do not correct the eigenvalues of $H^{\prime}$ with the perturbation term $H_{\delta}$, which does not exist. 
${ }^{1}$ E. Koutroumanou and A. K. Theophilou, preceding paper, Phys. Rev. B 34, 8004 (1986).

${ }^{2}$ E. Lagendijk and W. J. Huiskamp, Phyisca 65, 118 (1973).

${ }^{3}$ M. B. Schulz and C. D. Jeffries, Phys. Rev. 159, 277 (1967).

${ }^{4}$ E. J. Graeber, and G. H. Conrad, and S. F. Duliere, Acta Crystallogr. 21, 1012 (1966).

${ }^{5}$ E. Lagendijk, H. W. J. Blöte, and W. J. Huiskamp, Physica 61, 220 (1972).
${ }^{6}$ T. Niemeijer and H. W. J. Blöte, Physica 67, 125 (1973).

${ }^{7}$ J. M. Luttinger, and L. Tisza, Phys. Rev. 70, 954 (1946).

${ }^{8}$ J. Felsteiner, J. Phys. C 3, L174 (1970).

${ }^{9}$ S. K. Misra and J. Felsteiner, Phys. Rev. B 8, 1099 (1973).

${ }^{10}$ S. Thanos, and A. K. Theophilou, Phys. Rev. B 28, 1534 (1983).

11S. Thanos and A. K. Theophilou, Phys. Rev. B 28, 1544 (1983). 